\title{
Genetic variation in GABRB3 is associated with Asperger syndrome and multiple endophenotypes relevant to autism
}

\author{
Varun Warrier $^{1 *}$, Simon Baron-Cohen ${ }^{1,2}$ and Bhismadev Chakrabarti, ${ }^{1,3^{*}}$
}

\begin{abstract}
Background: Autism spectrum conditions (ASC) are associated with deficits in social interaction and communication, alongside repetitive, restricted, and stereotyped behavior. ASC is highly heritable. The gamma-aminobutyric acid (GABA)-ergic system has been associated consistently with atypicalities in autism, in both genetic association and expression studies. A key component of the GABA-ergic system is encoded by the GABRB3 gene, which has been previously implicated both in ASC and in individual differences in empathy.

Methods: In this study, 45 genotyped single nucleotide polymorphisms (SNPs) within GABRB3 were tested for association with Asperger syndrome (AS), and related quantitative traits measured through the following tests: the Empathy Quotient (EQ), the Autism Spectrum Quotient (AQ), the Systemizing Quotient-Revised (SQ-R), the Embedded Figures Test (EFT), the Reading the Mind in the Eyes Test (RMET), and the Mental Rotation Test (MRT). Two-loci, three-loci, four-loci haplotype analyses, and one seven-loci haplotype analysis were also performed in the AS case-control sample.

Results: Three SNPs (rs7180158, rs7165604, rs12593579) were significantly associated with AS, and two SNPs (rs9806546, rs11636966) were significantly associated with EQ. Two SNP-SNP pairs, rs12438141-rs1035751 and rs12438141-rs7179514, showed significant association with variation in the EFT scores. One SNP-SNP pair, rs7174437-rs1863455, was significantly associated with variation in the MRT scores. Additionally, a few haplotypes, including a $19 \mathrm{~kb}$ genomic region that formed a linkage disequilibrium (LD) block in our sample and contained several nominally significant SNPS, were found to be significantly associated with AS.
\end{abstract}

Conclusion: The current study confirms the role of GABRB3 as an important candidate gene in both ASC and normative variation in related endophenotypes.

Keywords: Asperger syndrome, Autism spectrum conditions, Empathy, Embedded Figures Test, Mental Rotation Test, GABA receptor

\section{Background}

Autism spectrum conditions (ASC) are neurodevelopmental, and are characterized by impairments in social interaction and communication alongside unusually narrow interests and repetitive behavior [1]. ASC are highly heritable, although environmental and epigenetic factors also

\footnotetext{
* Correspondence: vw260@medschl.cam.ac.uk; b.chakrabarti@reading.ac.uk 'Department of Psychiatry, Autism Research Centre, University of Cambridge, Cambridgeshire, UK

${ }^{3}$ Centre for Integrative Neuroscience and Neurodynamics, School of

Psychology and Clinical Language Sciences, University of Reading, Reading, UK

Full list of author information is available at the end of the article
}

contribute [2,3], and they affect $1 \%$ of the population [4]. Previous genetic research has largely focused on those individuals who also have learning difficulties. Asperger syndrome (AS) is a subgroup of ASC, and individuals with AS have no speech or cognitive delays [5].

Although no single gene accounts for all of the variation in the autism spectrum, several lines of evidence points to a common theme of neural development across genetic studies of autism. Genes involved in early brain development (including genes involved in synapse formation and stabilization, and those involved in neurotransmission

\section{Biomed Central}


in these early neural circuits) have been associated consistently with autism [6-8]. The gamma-aminobutyric acid (GABA)-ergic system plays a crucial role in early neural development. Both genetic association and gene expression studies suggest a key role for the GABA-ergic system in autism [9-11]. Specifically, variation in the GABRB3 gene in humans has been previously implicated in both empathy [12] and ASC [13-16]. The first study to associate variants in GABRB3 with ASC identified a statistically significant association between the marker 155CA-2 and individuals with ASC, using a multiple transmission disequilibrium test on 140 families [17]. This was replicated in 80 autism families [13]. Copy number variations and other chromosomal abnormalities at the GABRB3 locus have also been reported in individuals with ASC $[14,18]$.

Variation in GABRB3 is also associated with tactile sensitivity, which is atypical in some individuals with ASC [19]. Gabrb3 knockout mice exhibit deficits in social behavior and constitute a potential mouse model for autism [20]. GABRB3 encodes the B3 subunit of the $\mathrm{GABA}_{\mathrm{A}}$ receptor. $\mathrm{GABA}_{\mathrm{A}}$ receptor is an ionotropic, ligand-gated receptor, which is part of the inhibitory synapses in the adult brain, and selectively conducts $\mathrm{Cl}^{-}$ ions. During development, GABRB3 is an important molecule for neuronal growth and differentiation, and mediates excitatory signaling $[21,22]$.

In the present study we tested the importance of $G A B R B 3$ as a candidate gene in AS and in six potential endophenotypic measures of the autistic spectrum: three self-report measures (autistic traits, empathy, systemizing) and three performance measures (emotion recognition, attention to detail, and spatial processing).

The self-report measures used were as follows. The Autism Spectrum Quotient (AQ) is a measure of autistic traits. An AQ score above 32 is a predictor of ASC [23], and AQ scores show significant heritability in a general population sample [24]. Empathy was measured using the Empathy Quotient (EQ) [25]. On average, individuals with a diagnosis of AS will score significantly lower than controls on the EQ [25]. Several lines of research have suggested that individuals with ASC have difficulties with empathy, especially cognitive empathy [26]. Empathy in humans has a partly genetic basis $[27,28]$. Systemizing was measured using the Systemizing Quotient-Revised (SQ-R), in which individuals with AS score higher than controls $[27,28]$. Family genetic studies suggest that systemizing is part of the broader autistic phenotype in first-degree relatives of individuals with autism [29,30]. Although largely independent of each other, the EQ and the SQ-R predict scores on the AQ [31].

The performance measures were as follows. Complex emotion recognition ability was measured through the Reading the Mind in the Eyes test (RMET). This is an adult test of social sensitivity/cognitive empathy, in which individuals are shown photos of the eye region, and required to choose which word best represents what the person in the photograph is feeling or thinking [32]. On average, people with ASC score significantly lower than controls, and parents and siblings of people with ASC also show mild deficits in this test. Performance is normally distributed in the population, and scores on this test show a high degree of familiality [33]. Attention to detail was measured using the Embedded Figures Test (EFT), in which participants are asked to locate a target shape embedded within a complex pattern [34]. Individuals with ASC score above average [34], and scores on the EFT are also associated with high familiality [35]. Spatial processing was measured using the Mental Rotation Test (MRT), in which individuals are asked to process rotated pairs of letters and identify if the letter-pairs are identical to or mirror images of each other. Individuals with ASC perform better than controls on the MRT [36], and there is some evidence to suggest heritability of mental rotation ability [37].

To our knowledge, this is only the second study investigating the role of $G A B R B 3$ specifically in $A S$, and variations in EQ, AQ, EFT, and RMET scores. The first study (also from our group) found a genetic association between a variant in GABRB3 and empathy [12]. In the current study, we aimed to replicate and extend the findings of the first study in a new sample of volunteers. We hypothesized an association between the variants in GABRB3 with both AS and associated quantitative traits. Two separate experiments were conducted to test the role of GABRB3 in adults with AS. The first tested if any of 45 single nucleotide polymorphisms (SNPs) showed an association with a diagnosis of AS, using a case-control design. The second experiment tested for association of these SNPs with any of the quantitative endophenotypic measures described above, in the control population.

\section{Methods}

\section{Ethics approval}

The study was approved by the Cambridge Psychology Research Ethics Committee and by the NHS Research Ethics Committee (UK). All participants provided written consent to take part in this study. All research adhered to the Declaration of Helsinki.

\section{Participants}

All individuals involved in the current study were of Caucasian ancestry. Individuals free of any psychiatric or neurological disorders were recruited as controls by advertisements.

In total, 530 individuals participated in the case-control association study for AS. Of these, 412 (185 male, 227 female) individuals with an AQ score below 24 formed the controls, and 118 individuals with a DSM-IV (Diagnostic 
and Statistical Manual of Mental Disorders, Fourth Edition) or ICD-10 (International Classification of Diseases, Tenth Revision)-diagnosis of AS (74 male, 44 female) formed the cases. All cases were recruited through our online database, and were diagnosed with AS by independent clinicians (psychiatrists or clinical psychologists only) from established clinics. The mean AQ score of the cases was $35.6 \pm 8.9$. This is very close to the previously reported mean of $35.6 \pm 6.63$ for people with AS [21]. The mean AQ score in the general population is 16.4, with a standard deviation of 6.3 [38]. Taking this into consideration, we selected only individuals with an AQ score of below 24 as controls to ensure a balanced representation of individuals from two ends of the autistic trait continuum.

All the quantitative trait studies were conducted only in participants without a clinical diagnosis of AS. Individuals were invited to participate in online assessment of all six measures investigated in the study. In total, 412 individuals participated in the association study for the AQ, 413 for the EQ, and 414 for the SQ-R. The same individuals who were the controls in the case-control study participated in the association study for AQ. Additionally, 245 individuals participated in the association study for the MRT, REMT, and EFT.

Phenotypic scores for each measure and participant information are provided in Table 1.

\section{SNP selection}

In total, 45 SNPs were selected to provide maximal coverage through linkage disequilibrium (LD) of the GABRB3 gene region on chromosome 15 from 26802824 bp to 27200217 bp (GRCH37.p10 Primary Assembly, NCBI). SNPs were chosen to ensure that inter-SNP distance was less than $10 \mathrm{~kb}$. The choice of SNPs was constrained by the availability of the ABI TaqMan assays that were used for genotyping. Nine SNPs (rs12437672, rs1432007, rs12905535, rs737098, rs2114485, rs1426217, rs890317, rs7171512, rs8026392) are TagSNPs as indicated by the HapMap genome browser, release 27. Although the SNPs covered most of the genetic region, they did not completely flank the gene. Of the 45 SNPs, rs3212331 (Chr15: 27014769) is the most upstream, and rs2114485 is the most downstream (Chr15: 26802824) in the orientation of the gene. For the selected SNPs, the minor allele frequency (MAF) was above 0.05 in the CEU (Utah Residents with Northern and Western European Ancestry) population as calculated from the dbSNP database (http://www. ncbi.nlm.nih.gov/projects/SNP/). DNA was extracted from buccal samples, anonymized, and genotyped using the same protocol described in our first study [12]. No SNPs deviated significantly from Hardy-Weinberg equilibrium. Total genotyping rate across all the experiments was greater than $98 \%$.

\section{Association tests}

The two experiments were as follows.

\section{Case-control association study for AS}

SNP association tests were conducted for all SNPs on all cases with AS $(n=118)$, and controls $(n=412$, with an AQ score below 24). SNP-SNP interaction tests were then carried out on this sample. In total, 990 possible SNP-SNP combinations $(45 \times 45)$ were tested for association using logistic regression, and significant SNPs were additionally checked for LD between them. Interactions were reported to be significant if the SNP pairs were not in LD.

Table 1 Phenotype measures and participant information

\begin{tabular}{|c|c|c|c|c|c|c|c|c|}
\hline \multirow[t]{2}{*}{ Participants } & \multicolumn{2}{|c|}{ Case-control } & \multirow[t]{2}{*}{$\mathrm{AQ}^{\mathrm{a}}$} & \multirow[t]{2}{*}{ EQ } & \multirow[t]{2}{*}{ SQ-R } & \multirow[t]{2}{*}{ RMET $^{\mathbf{b}}$} & \multirow[t]{2}{*}{$\mathrm{EFT}^{\mathrm{b}}$} & \multirow[t]{2}{*}{$\mathrm{MRT}^{\mathrm{b}}$} \\
\hline & Cases & Controls $^{a}$ & & & & & & \\
\hline \multicolumn{9}{|l|}{ Numbers } \\
\hline Total & 118 & 412 & 412 & 413 & 414 & 245 & 245 & 245 \\
\hline Male & 74 & 185 & 185 & 209 & 210 & 122 & 122 & 122 \\
\hline Female & 44 & 227 & 227 & 204 & 204 & 123 & 123 & 123 \\
\hline \multicolumn{9}{|c|}{ Trait score, mean $\pm S D$} \\
\hline Overall & $35.6 \pm 8.9^{c}$ & $14.9 \pm 5.0^{c}$ & $14.9 \pm 5.0$ & $41.5 \pm 12.3$ & $60.3 \pm 20.6$ & $27.1 \pm 3.2$ & $11.6 \pm 1$ & $16.8 \pm 2.6$ \\
\hline Male & $35.1 \pm 8.7^{c}$ & $16.0 \pm 4.4^{c}$ & $16.0 \pm 4.4$ & $36.8 \pm 12.4$ & $63.9 \pm 20.0$ & $27.06 \pm 3.2$ & $11.7 \pm 0.6$ & $17.4 \pm 2.1$ \\
\hline Female & $36.6 \pm 8.8^{c}$ & $13.9 \pm 5.1^{c}$ & $13.9 \pm 5.1$ & $45.6 \pm 12.3$ & $56.5 \pm 20.4$ & $27.1 \pm 3.2$ & $11.6 \pm 1.2$ & $16.1 \pm 2.9$ \\
\hline Range & 7 to 50 & 2 to 23 & 2 to 23 & 2 to 24 & 11 to 139 & 17 to 34 & 2 to 12 & 7 to 20 \\
\hline
\end{tabular}

Abbreviations: AQ, Autism Spectrum Quotient; EFT, Embedded Figures Test; EQ, Empathy Quotient; MRT, Mental Rotation Test; RMET, Reading the Mind in the Eyes Test; SQ-R, Systemizing Quotient, Revised.

${ }^{a}$ The same individuals participated in the case-control and $A Q$ quantitative trait studies.

${ }^{\mathrm{b}}$ The same individuals participated in REMT, EFT, and MRT.

${ }^{\text {'T } T r a i t ~ s c o r e s ~ f o r ~ c a s e-c o n t r o l ~ s t u d y ~ a r e ~ t h e ~ A d u l t ~ A u t i s m ~ S p e c t r u m ~ Q u o t i e n t ~ s c o r e s . ~}$ 


\section{Quantitative trait association study for AS}

Quantitative trait association studies were performed for the AQ, SQ-R, EQ, ET, EFT and MRT. SNP association tests on each of these quantitative traits were conducted in controls only. The number of participants in each experiment differed (Table 1). SNP-SNP interaction tests were tested for association with each of the six quantitative traits, for a total of 990 SNP combinations, and modeled using linear regression. As in the previous experiment, interactions were reported to be significant if the SNP pairs were not in LD.

\section{Statistical analysis}

All statistical analyses were performed using Plink version 1.07 (http://pngu.mgh.harvard.edu/ purcell/plink/) [39]. Bonferroni correction was implemented to correct for multiple SNPs and phenotypes tested. A threshold of significance $(\alpha)$ was calculated after both the corrections. $P$-values were reported as significant if they were below the $\alpha$ threshold.

The effective number of independent SNPs was estimated using SNPSpD (http://genepi.qimr.edu.au/general/daleN/ SNPSpD/) [40]. For the SNP association studies the total number of independent loci (after taking into account the LD between SNPs) and the new $\alpha$ value was determined for each test using SNPSpD. On average, the total number of independent loci was calculated to be 30.5 , although this varied marginally between tests (because of small differences in the number of individuals who completed each quantitative measure). For SNP-SNP interaction, a total of 990 possible SNP-SNP combinations was tested. However, SNP-SNP interaction can be calculated accurately only for independent loci. The total number of independent SNP-SNP combinations was thus determined to be $465(31 \times 30) / 2)$. The Bonferroni corrected $\alpha$ was set to 0.000108 .

Bonferroni correction was also used to correct for the multiple phenotypes tested. Owing to differences in samples for the phenotypes tested the experiments were broadly divided into three groups for correction.

Group 1 was the case-control association test for AS, which had the largest sample size and was distinct because of inclusion of cases (Experiment 1). In this sample, only one phenotype (that is, clinical diagnosis) was tested, and hence correction for multiple phenotypes was not implemented.

Experiment 2 consisted of two largely distinct samples. The first of these samples, group 2, comprised the AQ, EQ and SQ-R, as more than three-quarters of the participants had participated in two or more of these tests. However, as all three phenotypes had a high degree of correlation between them, these were not treated as truly independent observations. Because Bonferroni correction tends to be over-corrective when traits are correlated, we did not use this for multiple phenotypes in this group.

The second sample within experiment 2, group 3, comprised the three performance-based tests (the RMET, EFT, and MRT) and was a subsample of group 2.

In total, 245 individuals took part in all three tests. There was little correlation between the phenotype scores for these tests in our sample. Bonferroni correction was implemented for this group. The $\alpha$ value for SNP association tests and SNP-SNP interaction tests derived from SNPSpD was divided by 3 to get the final $\alpha$ value. For SNP-SNP interaction tests on the performance-based tests, the final $\alpha$ value was 0.000036 (or 0.000108 divided by 3).

\section{Haplotype analysis}

Haplotype analysis was performed for case-control AS dataset using Plink. Haplotypes were phased using the E-M algorithm, and global or omnibus two-loci, three-loci, and four-loci haplotype analyses were performed using logistic regression on Plink. Additionally, one large LD block, which is $19 \mathrm{~kb}$ long from rs7174437 to rs7180158, was considered for haplotype analysis. There are nine SNPs located in this region, including all three significant SNPs and three nominally significant SNPs for the case-control AS association study. $P$-values were corrected for family-wise error rates using permutation correction with 50,000 permutations, and the $\alpha$ value for significance was kept at 0.05 .

\section{LD analyses and SNP annotation}

LD values between SNPs of interest in the HapMap CEU population data were calculated using SNAP (http://www.broadinstitute.org/mpg/snap/) [41]. LD values between SNPs in our sample were calculated using Plink. $L D$ values have been reported using $R^{2}$ rather than $D^{\prime}$ as $R^{2}$ takes into account allele frequencies in populations. LD plots for the sample studied were created using Haploview [42].

Integrated putative functional scores for the SNPs tested were obtained using both the F-SNP server (http://compbio.cs.queensu.ca/F-SNP) [43] and FastSNP (http://fastsnp.ibms.sinica.edu.tw/pages/input_Candidate GeneSearch.jsp) [44]. SNPnexus (http://www.snp-nexus. org/) [45] was used to investigate SNP conservation and structural variants in the locus investigated. Haploreg (http://www.broadinstitute.org/mammals/haploreg/) [46] was used to check for histone markers, conservation scores, and DNAse hypersensitivity sites for the SNP of interest and other SNPs in high LD $\left(\mathrm{R}^{2}>0.8\right)$. This was also manually cross-checked using the UCSC Genome Browser (http://genome.ucsc.edu/) [47]. Finally, the Genetic Association Database (http://geneticassociationdb.nih. gov/) [48] was used to check for reports of any previous associations for the significant SNPs. 
Table 2 GABRB3 SNP association results for AS and the six quantitative endophenotypes investigated

\begin{tabular}{|c|c|c|c|c|c|c|c|c|c|}
\hline \multirow[t]{2}{*}{ SNP } & \multirow[t]{2}{*}{ SNP position } & \multicolumn{2}{|c|}{ AS case-control } & \multicolumn{6}{|l|}{$P$ value } \\
\hline & & OR & $P$ value & $A Q$ & EQ & SQ-R & EFT & REMT & MRT \\
\hline rs2114485 & 26802824 & 0.9369 & 0.704 & 0.6105 & 0.6514 & 0.6369 & 0.6719 & 0.1518 & 0.9454 \\
\hline rs11631940 & 26806814 & 0.8902 & 0.5088 & 0.6759 & 0.8435 & 0.1997 & 0.8645 & 0.8271 & 0.4787 \\
\hline rs1432007 & 26810689 & 1.127 & 0.4205 & 0.7813 & 0.6526 & 0.5193 & 0.7481 & 0.2376 & 0.7725 \\
\hline rs1426217 & 26821125 & 1.082 & 0.5975 & 0.439 & 0.4008 & 0.5922 & 0.5827 & 0.6484 & 0.5826 \\
\hline rs10519563 & 26823404 & 1.096 & 0.6204 & 0.8042 & 0.1096 & 0.1448 & 0.3941 & 0.9824 & 0.2969 \\
\hline rs12440905 & 26826281 & 1.584 & 0.3943 & 0.9656 & 0.2318 & 0.9096 & 0.5663 & 0.6207 & 0.5445 \\
\hline rs17646555 & 26837651 & 1.089 & 0.749 & 0.7359 & 0.0351 & 0.7584 & 0.6143 & 0.2616 & 0.1865 \\
\hline rs12437672 & 26838405 & 1.016 & 0.9246 & 0.6538 & 0.3636 & 0.4328 & 0.8751 & 0.675 & 0.4629 \\
\hline rs1582760 & 26853487 & 1.114 & 0.6609 & 0.902 & 0.2335 & 0.7375 & 0.4951 & 0.4093 & 0.3209 \\
\hline rs2873027 & 26867409 & 0.9804 & 0.8947 & 0.4164 & 0.7438 & 0.4436 & 0.3401 & 0.996 & 0.7369 \\
\hline rs12438141 & 26870602 & 1.094 & 0.6625 & 0.2593 & 0.05641 & 0.7694 & 0.04683 & 0.1429 & 0.004928 \\
\hline rs10873636 & 26888978 & 0.8377 & 0.2985 & $0.01893^{a}$ & $0.01765^{\mathrm{a}}$ & $0.04946^{a}$ & 0.8912 & 0.05818 & 0.02446 \\
\hline rs11636966 & 26893028 & 0.9915 & 0.9547 & 0.06219 & $0.000594^{a}$ & 0.8307 & 0.7713 & 0.5205 & 0.7296 \\
\hline rs9806546 & 26893386 & 0.7572 & 0.188 & 0.06427 & 0.001469 & $0.009834^{a}$ & 0.6132 & 0.4449 & 0.3145 \\
\hline rs8023959 & 26894305 & 1.034 & 0.887 & 0.1065 & 0.7408 & 0.8473 & 0.3852 & 0.05979 & 0.06356 \\
\hline rs7179514 & 26895324 & 1.028 & 0.8525 & 0.107 & $0.002318^{a}$ & 0.5474 & 0.3509 & 0.5521 & 0.1739 \\
\hline rs7171512 & 26906345 & 0.9611 & 0.7976 & 0.3705 & $0.01302^{a}$ & 0.3096 & 0.7664 & 0.1414 & 0.9829 \\
\hline rs12442889 & 26917100 & 1.041 & 0.8066 & 0.3348 & 0.2381 & 0.3188 & 0.8606 & 0.6945 & 0.1025 \\
\hline rs1367959 & 26917158 & 1.178 & 0.315 & 0.7905 & $0.001742^{a}$ & 0.1215 & 0.02745 & 0.2206 & 0.05204 \\
\hline rs1863455 & 26918169 & 0.8474 & 0.4769 & 0.2206 & 0.5058 & 0.4483 & 0.3591 & 0.005442 & 0.8047 \\
\hline rs890317 & 26922201 & 0.8293 & 0.2741 & 0.9942 & 0.1927 & 0.9031 & 0.09281 & 0.887 & 0.8351 \\
\hline rs11161329 & 26926710 & 0.8283 & 0.2203 & 0.5383 & 0.07182 & 0.6331 & 0.02263 & 0.07253 & 0.7137 \\
\hline rs12593482 & 26933545 & 1.119 & 0.7621 & 0.7125 & $0.04769^{\mathrm{a}}$ & 0.2482 & 0.6505 & 0.9041 & 0.6601 \\
\hline rs1035751 & 26943796 & 0.8033 & 0.4228 & 0.531 & 0.8884 & 0.9924 & 0.1161 & 0.9053 & 0.4785 \\
\hline rs7181473 & 26950087 & 1.018 & 0.9072 & 0.4267 & 0.5739 & 0.9399 & 0.7201 & 0.8816 & 0.6508 \\
\hline rs17117279 & 26952672 & 0.8777 & 0.5218 & 0.3016 & 0.09375 & 0.6948 & 0.1173 & 0.2397 & 0.4522 \\
\hline rs1426224 & 26953091 & 0.984 & 0.9632 & 0.3725 & 0.1801 & 0.2826 & 0.5186 & 0.1248 & 0.4864 \\
\hline rs1549482 & 26953764 & 1.073 & 0.7015 & 0.9659 & 0.6341 & 0.6026 & 0.072 & 0.4522 & 0.3501 \\
\hline rs4906896 & 26960426 & 1.354 & $0.04067^{a}$ & $0.001682^{a}$ & $0.01946^{a}$ & 0.8831 & 0.05366 & $0.03463^{a}$ & 0.126 \\
\hline rs737098 & 26967170 & 0.7071 & $0.04886^{a}$ & 0.1189 & 0.1006 & $0.03769^{a}$ & 0.9156 & 0.5102 & 0.6054 \\
\hline rs2315904 & 26967522 & 0.8293 & 0.4539 & 0.4212 & 0.5888 & 0.08328 & 0.1348 & 0.3303 & 0.1788 \\
\hline rs1863456 & 26974032 & 1.006 & 0.9703 & 0.116 & 0.06622 & 0.7006 & 0.2814 & 0.6925 & 0.271 \\
\hline rs2162241 & 26976782 & 0.7075 & $0.02707^{a}$ & 0.2351 & 0.1778 & 0.2413 & 0.5519 & 0.5273 & 0.8923 \\
\hline rs7180158 & 26978238 & 2.15 & $0.000358^{\mathrm{a}}$ & 0.4416 & 0.2842 & $0.01907^{a}$ & 0.3974 & 0.7486 & 0.124 \\
\hline rs $8026932^{b}$ & 26978906 & 1.263 & 0.5769 & $0.006922^{a}$ & 0.9863 & 0.7881 & 0.6083 & 0.1999 & 0.9726 \\
\hline rs $6576602^{b}$ & 26982080 & 1.46 & $0.03347^{a}$ & 0.189 & 0.3375 & $0.00998^{a}$ & 0.05174 & 0.4184 & $0.01776^{\mathrm{a}}$ \\
\hline rs890318 & 26982378 & 0.745 & 0.2896 & 0.2458 & 0.8865 & 0.3377 & 0.08322 & 0.4733 & 0.1141 \\
\hline rs12593579 b & 26988132 & 1.96 & $0.000668^{a}$ & 0.5066 & 0.335 & $0.03631^{a}$ & 0.3711 & 0.74 & 0.2056 \\
\hline rs $8038471^{b}$ & 26990651 & 1.154 & 0.3372 & 0.08613 & $0.01078^{a}$ & 0.9937 & 0.3891 & 0.8799 & 0.6707 \\
\hline rs12905535 & 26992166 & 0.6169 & $0.005747^{\mathrm{a}}$ & 0.08597 & $0.03931^{a}$ & $0.04997^{a}$ & 0.283 & 0.4318 & 0.07415 \\
\hline rs7165604 & 26994456 & 2.452 & $0.000451^{\mathrm{a}}$ & 0.1189 & 0.1297 & 0.1982 & 0.278 & 0.8782 & 0.09043 \\
\hline rs $7174437^{b}$ & 26997923 & 2.022 & $0.01567^{\mathrm{a}}$ & 0.1185 & $0.02142^{a}$ & 0.5118 & 0.4765 & 0.8321 & 0.4211 \\
\hline rs7178713 & 27002886 & 0.6789 & $0.01877^{\mathrm{a}}$ & 0.4842 & 0.1292 & 0.1468 & 0.5308 & 0.1201 & 0.1008 \\
\hline
\end{tabular}


Table 2 GABRB3 SNP association results for AS and the six quantitative endophenotypes investigated (Continued)

\begin{tabular}{llllllllll}
\hline rs8026392 & 27008723 & 0.8825 & 0.4244 & 0.05513 & $0.02792^{\mathrm{a}}$ & 0.4595 & 0.4105 & 0.6825 & 0.3217 \\
rs3212331 & 27014769 & 0.896 & 0.53 & 0.6579 & 0.7116 & 0.6821 & 0.2821 & 0.8648 & 0.5415 \\
\hline
\end{tabular}

Abbreviations: AQ Autism Spectrum Quotient, EFT Embedded Figures Test, EQ Empathy Quotient, MRT Mental Rotation Test, RMET Reading the Mind in the Eyes Test, SQ- $R$ Systemizing Quotient, Revised, SNP single nucleotide polymorphism.

${ }^{a}$ Nominally significant $P$-values $(P<0.05)$.

${ }^{b}$ SNPs found in haplotype block 7 (as referred to in Figure 2).

\section{Results}

\section{SNP associations}

\section{Case-control association}

Ten SNPs were nominally associated with AS (Table 2). Of these, rs7180158, rs7165604, and rs12593579 survived Bonferroni correction (Table 3). All three SNPs are located in the intron 4 of the GABRB3 gene (Figure 1). In our sample, all three SNPs were found to be a part of an LD block as calculated by Haploview (Figure 2). Nine SNPs form the LD block. Three of the SNPS (rs7180158, rs7165604, and rs12593579) were significant after Bonferroni correction and another three of these SNPs (rs12905535, rs7174437, rs6576602) were nominally significant in the AS association study. For the LD structure for GABRB3 in the HapMap CEU population, see Additional file 1. There were no significant SNP-SNP interactions for the case-control analysis (see Additional file 2).

Table 3 Significant haplotypes for case-control (Asperger syndrome)

\begin{tabular}{|c|c|c|}
\hline $\begin{array}{l}\text { No of } \\
\text { SNPs }\end{array}$ & SNP haplotype & $\begin{array}{l}\text { FWER corrected } \\
P \text {-value }\end{array}$ \\
\hline 2 & rs1863456-rs2162241 & 0.03284 \\
\hline 2 & rs2162241-rs7180158 & 0.03882 \\
\hline 2 & rs7180158-rs8026932 & 0.0304 \\
\hline 2 & rs6576602-rs890318 & 0.03576 \\
\hline 2 & rs890318-rs8038471 & 0.021 \\
\hline 2 & rs12593579-rs8038471 & 0.00582 \\
\hline 2 & rs12905535-rs7165604 & 0.01768 \\
\hline 3 & rs4906896-rs737098-rs2315904 & 0.02002 \\
\hline 3 & rs1863456-rs2162241-rs7180158 & 0.02438 \\
\hline 3 & rs8026932-rs6576602-rs890318 & 0.0209 \\
\hline 3 & rs890318-rs12593579-rs8038471 & 0.0145 \\
\hline 3 & rs12593579-rs8038471-rs12905535 & 0.01192 \\
\hline 3 & rs8038471-rs12905535-rs7165604 & 0.03052 \\
\hline 4 & rs7180158-rs8026932-rs6576602-rs890318 & 0.03832 \\
\hline 4 & rs8026932-rs6576602-rs890318-rs12593579 & 0.04774 \\
\hline 4 & rs6576602-rs890318-rs12593579-rs8038471 & 0.02428 \\
\hline 4 & rs890318-rs12593579-rs8038471-rs12905535 & 0.01002 \\
\hline 4 & rs12593579-rs8038471-rs12905535-rs7165604 & 0.0454 \\
\hline 9 & $\begin{array}{l}\text { rs7180158-rs8026932-rs6576602-rs890318- } \\
\text { rs12593579-rs8038471-rs12905535- } \\
\text { rs7165604-rs7174437 }\end{array}$ & 0.00534 \\
\hline
\end{tabular}

Abbreviations: FWER family-wise error rate, SNP single nucleotide polymorphism.

\section{Quantitative trait associations}

Two SNPs were nominally associated with the RMET, seven were nominally associated with the SQ-R, and 13 were nominally associated with the EQ, while three SNPs each were nominally associated with the AQ, EFT, and MRT (Table 2). Two SNPs, rs11636966 and rs9806546, survived Bonferroni correction for the EQ (see Table 4); both of these SNPs are located in intron 5 of the GABRB3 gene (Figure 1). No SNP was nominally significant across all the six measures. Two SNP-SNP interactions were found to be significantly associated with the EFT after Bonferroni correction (Table 4). These were: rs12438141rs1035751 $\left(\mathrm{R}^{2}=0.017\right)$ and rs12438141-rs7179514 $\left(\mathrm{R}^{2}=0.001\right)$ (Table 4, Figure 1). One SNP-SNP interaction, rs7174437-rs1863455, was found to be significant for the MRT $\left(R^{2}=0.003\right)$ (Table 4, Figure 1). Neither of the SNPs was nominally significant in the SNP association study for the MRT. There were no significant SNP-SNP interactions for any of the other traits tested (see Additional file 3).

\section{Haplotype analysis}

Seven two-loci haplotypes, six three-loci haplotypes, five four-loci haplotypes, and one nine-loci haplotype, which spanned a 19 kb entire LD block (rs7180158-rs7174437; Chr15:26978238-26997923), were significant after permutation correction (Table 3; see Additional file 4). All the haplotypes except two two-loci haplotypes [rs1863456-rs2162241 (Chr15: 26974032-26976782) and rs2162241-rs7180158 (Chr15: 26976782-26978238)] and two three-loci haplotypes (rs4906896-rs737098-rs2315904 (Chr15: 26960426-26967522) and rs1863456-rs2162241rs7180158 (Chr15: 26974032-26978238)) are part of the $19 \mathrm{~kb}$ region. The four haplotypes not a part of this region lie immediately upstream (see Figure 2) The omnibus nine-loci haplotype had a corrected $P$-value of 0.00534 , indicating that the genomic region as a whole (Figure 2) is associated with AS in our sample.

\section{Discussion}

In this study, we tested multiple common variants in GABRB3 for association with AS and related endophenotypes in a general population sample. To our knowledge, this is the first study to incorporate such a comprehensive group of phenotypes (including clinical diagnosis, self-report questionnaires, and observational measures on both social and non-social aspects of autistic cognition). 


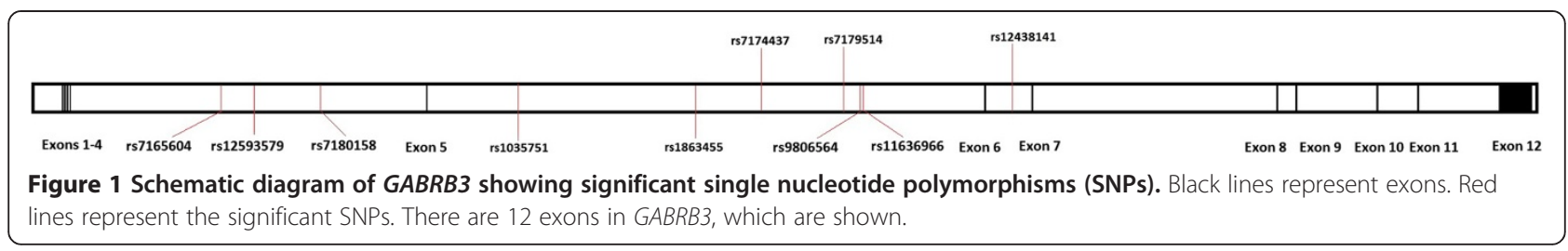

Nine SNPs were nominally associated with AS, of which three (rs7180158, rs7165604, rs12593579) retained their significance after Bonferroni correction. All three SNPs are located in a $19 \mathrm{~kb}$ genomic region that forms an $\mathrm{LD}$ block in our sample. The entire block was found to be associated with AS when tested for global haplotype association. For the EQ, thirteen SNPs were nominally significant, of which two (rs9806546, rs11636966) remained significant after Bonferroni correction. Two SNP-SNP interactions were significant for the EFT (rs12438141rs1035751 and rs12438141-rs7179514) and one for the MRT (rs7174437-rs1863455) after Bonferroni correction. None of the SNPs was located in regions of common structural variations, $\mathrm{CpG}$ islands, or affected micro RNA binding sites, as calculated by SNPnexus and the UCSC Genome Browser.

The three SNPs that were significant in AS case-control studies are located in intron 4, within a region of 16,218 base pairs. In our sample, they were found to be a part of a shared $19 \mathrm{~kb}$ LD block, which is also associated with AS. This genomic region extends from rs7180158 (Chr15: 26978238, dbSNP) to rs7174437 (Chr15: 26997923, dbSNP) in intron 4, and is 19,905 bp downstream of exon 3 and 16,326 bp upstream of exon 4. When queried in FastSNP, rs12593579 was suggested to disrupt an enhancer region. Both rs7165604 and rs12593579 are found in putative transcription regulatory sites according to F-SNP. Further, according to Haploreg, these SNPs disrupt transcription factor binding sites either directly or through common variants in high LD. The two SNPs significantly associated with the EQ are located in intron 5, and are a mere $358 \mathrm{bp}$ apart. However, they are not in significant LD either in the CEU population $\left(R^{2}: 0.182\right.$; see Additional file 1: Table S1) or in our sample $\left(\mathrm{R}^{2}=0.157\right)$ (see Additional file 1: Figure $\mathrm{S} 2$ ). It is hence likely that the effect of the SNPs have been independently measured, although this does not

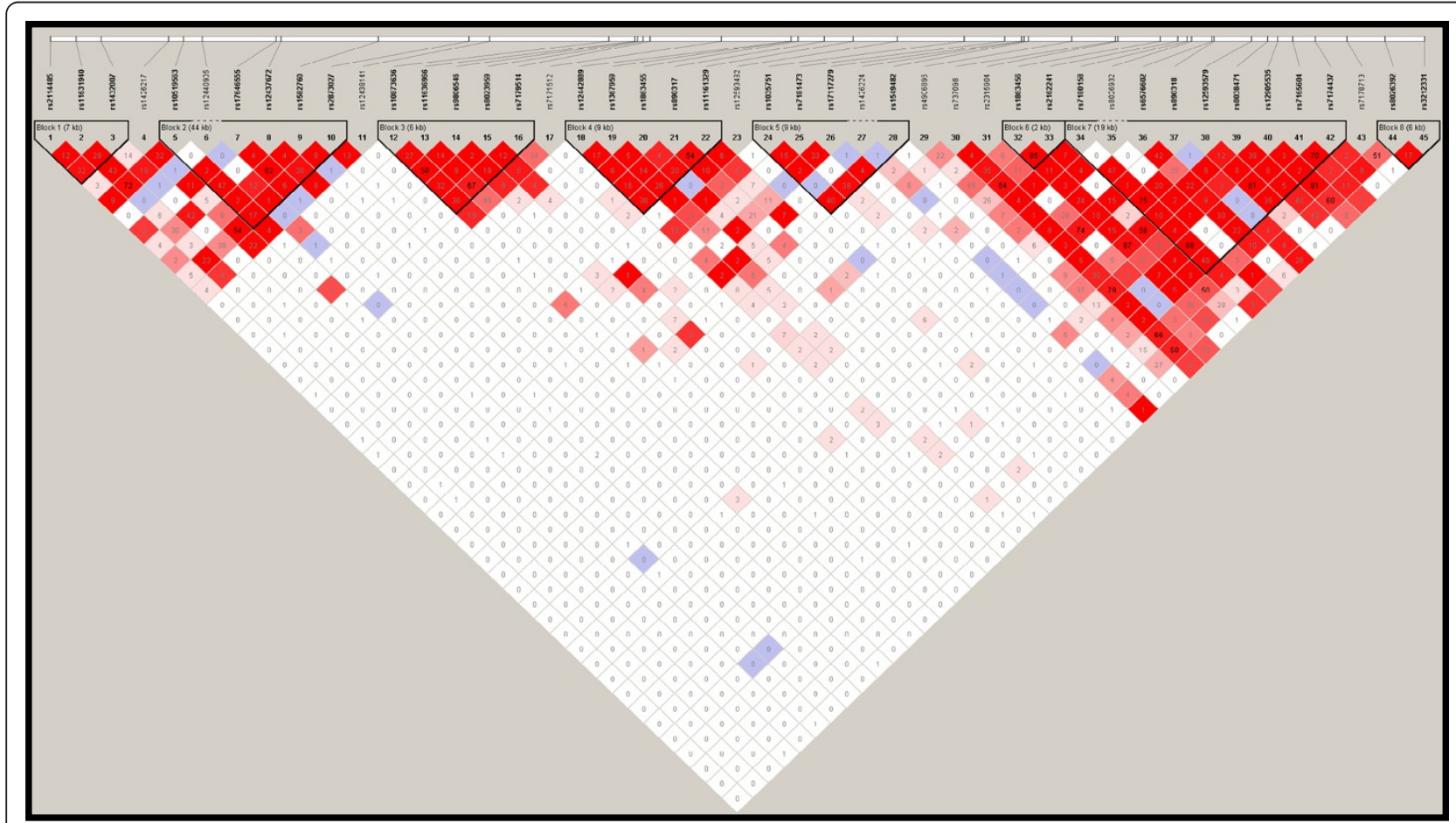

Figure 2 Linkage disequilibrium (LD) structure of GABRB3 for the Asperger syndrome (AS) case-control dataset. LD structure of the region investigated in the AS case-control dataset (412 controls and 118 cases), as plotted on Haploview is shown. In this graph, 45 single nucleotide polymorphisms (SNPs) that were genotyped have been plotted. They fall into eight LD blocks. 
Table 4 Significant SNPs and SNP-SNP pairs

\begin{tabular}{|c|c|c|c|c|c|c|c|}
\hline \multirow{2}{*}{$\begin{array}{l}\text { SNP or SNP-SNP } \\
\text { interaction }\end{array}$} & \multirow[t]{2}{*}{ Phenotype } & \multirow{2}{*}{$\begin{array}{l}\text { P values } \\
\text { (uncorrected) })^{a}\end{array}$} & \multirow{2}{*}{$\begin{array}{l}\text { Minor allele/major } \\
\text { allele }^{\mathbf{b}}\end{array}$} & \multirow{2}{*}{$\begin{array}{l}\text { MAF (HapMap } \\
\text { CEPH)c }\end{array}$} & \multicolumn{2}{|c|}{ Frequency of minor allele } & \multirow[t]{2}{*}{$x^{2}$} \\
\hline & & & & & Cases & Controls & \\
\hline rs7180158 & AS & $3.58 \mathrm{E}-04$ & $A / G$ & $A=0.24$ & 0.1624 & 0.08272 & 12.74 \\
\hline rs7165604 & AS & $4.51 \mathrm{E}-04$ & $C / T$ & $C=0.17$ & 0.1174 & 0.05145 & 12.31 \\
\hline rs12593579 & AS & $6.68 \mathrm{E}-04$ & $C / A$ & $C=0.21$ & 0.194 & 0.1093 & 11.58 \\
\hline rs11636966 & EQ & 5.94E-04 & $\mathrm{T} / \mathrm{C}$ & $\mathrm{T}=0.22$ & NA & NA & NA \\
\hline rs9806546 & EQ & 1.47E-03 & $A / G$ & $A=0.40$ & NA & NA & NA \\
\hline rs12438141-rs1035751 & EFT & 7.59E-06 & $C / T-A / G$ & $T=0.13, G=0.06$ & NA & NA & NA \\
\hline rs12438141-rs7179514 & EFT & $5.21 \mathrm{E}-06$ & $C / T-C / G$ & $\mathrm{~T}=0.13, \mathrm{G}=0.31$ & NA & NA & NA \\
\hline rs7174437-rs1863455 & MRT & $1.58 \mathrm{E}-05$ & $C / T-C / T$ & $C=0.12, C=0.08$ & NA & NA & NA \\
\hline
\end{tabular}

Abbreviations: AQ, Autism Spectrum Quotient; AS, Asperger syndrome; EFT, Embedded Figures Test; EQ, Empathy Quotient; MRT, Mental Rotation Test; NA, not applicable; SNP, single nucleotide polymorphism.

${ }^{a}$ Corrected P-value threshold is $3.6 \times 10^{-05}$.

${ }^{\mathrm{b}}$ Alleles for SNP-SNP pairs have been provided in the order they are written.

${ }^{\mathrm{C}} \mathrm{MAF}$ for SNP-SNP pairs have been provided in the order they are written.

exclude the possibility that a single non-tested variant situated close by is contributing to the effect seen at the two SNPs. Genotyping all the variants in this short region and testing them for association with the EQ should be undertaken in a future study. The most significantly associated SNP rs11636966 markedly alters an En-1_2 binding site (Haploreg). There is considerable evidence to suggest a role for proteins of the Engrailed family in early neurodevelopment and ASC [49-52]. Both these SNPs are also in high LD with several SNPs that putatively alter other transcription factor binding sites. A previous study from our laboratory [12] tested for association between two SNPs in GABRB3 (rs2873027 and rs11161335) with both AS and the EQ. Neither of the two SNPs was significantly associated with AS, but rs 2873027 was found to be associated with the EQ. In the current study, only rs2873027 was included in the 45 SNPs genotyped. rs2873027 was not significantly associated with the EQ in the current study.

Two SNP-SNP interactions were found to contribute to variation in the EFT scores. Three SNPs participate in these interactions and none of them is in significant $\mathrm{LD}$ with each other either in the CEU population sample or in our sample (see Additional file 1: Table S1; see Additional file 1: Figure S3). Two of these SNPs, rs7179514 and rs1035751, are located in intron 5, while rs12438141 is located in intron 6. According to both FastSNP and F-SNP, rs1035751 and rs12438141 alter transcription regulatory sites. All SNPs except rs1035751 disrupt regulatory motifs (Haploreg). A single SNP-SNP interaction, rs1863455-rs7174437, was significantly associated with the MRT. rs1863455 is located in intron 5 and rs7174437 in intron 4, and they are 79,754 bp apart. They are not in significant $L D\left(R^{2}=0.003\right)$ (see Additional file 1: Table S1) in the CEU population, and are not a part of an LD block in our sample (see Additional file 1: Figure S3).
Both the SNPs disrupt regulatory motifs (FastSNP, F-SNP, and Haploreg). rs7174437 disrupts regulatory motifs of the Sox family of transcription factors (Haploreg), which play a pivotal role during development [53].

The putative functional annotation of the SNPs indicates that a number of the significantly associated SNPs alter GABRB3 transcription during and after embryonic development either directly or via other variants that are in high LD. Of the ten SNPs found to be significant, three are in intron 4, six in intron 5, and one in intron 6 . GABRB3 has twelve exons (see Figure 1) and four transcript variants in NCBI. Transcript variants 1 and 2 are more common at a cellular level, but neither of them have exons 5 and 6 . Transcript variant 3 , which has been described only at the transcript level, starts from exon 5, whereas transcript variant 4 , which has been described at the protein level, starts from exon 6 . There are currently no studies describing the function of these transcripts. It is possible that the variants in introns 4 and 5 act to regulate levels of these alternate transcripts. Alternatively, the variants could also act as intronic regulatory elements to alter the levels of transcript variants 1 and 2. Cellular studies need to be performed to validate either of these hypotheses.

We did not replicate the exact SNP association reported by Chakrabarti et al. [12]. However, the inference in our earlier paper was at the gene level (that is, the statistical inference from the permutation analysis was that one or more SNPs in the gene were significantly associated with the phenotype, and not specifically for any SNP). The current study replicates the association of GABRB3 with empathy at the gene level, and provides evidence for the association of GABRB3 with AS, the EFT, and the MRT.

A limitation of this study is the moderate sample size. Replication of the results in a larger sample size is required to validate the findings. Although no SNP was significant 
across two or more traits, the experiments were not completely independent as several individuals participated in two or more experiments. Additionally, the low variance in the phenotypic scores of EFT must be taken into account when interpreting the results. To assess if the limited variance was restricted to the genotyped sample, we checked for EFT scores in a larger database. Both the mean EFT score and standard deviation were similar between the two datasets, indicating that the low variance in the EFT scores in our sample was not an artifact. Although none of the cases studied were formally tested for IQ, an essential feature for the clinical diagnosis of AS is that individuals diagnosed as having AS or high-functioning autism (as was the case for the ASC sample in our study) is that individuals have an IQ in the average range [54]. Average range is defined as two standard deviations either side of the mean, so this would mean an IQ of over 70. Further, it has been shown that IQ and autistic traits show limited genetic covariance [55]. It is therefore reasonable to infer that IQ differences are unlikely to confound the conclusions drawn from the current study. Finally, it is important to remember that $G A B R B 3$ is not the sole gene that contributes to either AS or the traits tested, and that other genes could act either independently of or epistatically with $G A B R B 3$ to contribute to these complex phenotypes.

\section{Conclusions}

We report novel associations of SNPs in GABRB3 with AS, and normative variation in empathy. We did not find any allelic association between SNPs in GABRB3 and the five other endophenotypes tested. A large $19 \mathrm{~kb}$ LD block was also positively associated with AS in our sample. We also found three intragenic SNP-SNP interactions that are significantly associated with two of the endophenotypes. Two SNP-SNP interactions were associated with EFT, and one SNP-SNP interaction is associated with MRT. This is the first study to specifically test the association of such a large number of SNPs in $G A B R B 3$ with AS, along with a comprehensive group of endophenotypes.

\section{Additional files}

Additional file 1: Linkage disequilibrium (LD) information for the single nucleotide polymorphisms (SNPs) investigated. A table presenting pairwise LD for the significant GABRB3 SNPS genotyped in the HapMap CEU population. Three LD plots for the region investigated in 1) the CEU population; 2) the cohort investigated for the Empathy Quotient (EQ); 3) the cohort investigated for the Embedded Figures Test (EFT), Mental Rotation Test (MRT) and Reading the Mind in the Eyes Test (RMET).

Additional file 2: GABRB3 SNP-SNP interaction results for case-control (AS). AS, Asperger syndrome; SNP, Single nucleotide polymorphism.
Additional file 3: GABRB3 SNP-SNP interaction results ( $P$-values) for the six quantitative traits investigated. SNP, Single nucleotide polymorphism.

Additional file 4: GABRB3 case-control (AS) results for haplotype analysis. AS, Asperger syndrome.

\section{Abbreviations}

AQ: Autism spectrum quotient; AS: Asperger syndrome; ASC: Autism spectrum conditions; EFT: Embedded figures test; EQ: Empathy quotient; GABA: Gamma-aminobutyric acid; GABRB3: Gamma-aminobutyric acid receptor subunit beta-3; LD: Linkage disequilibrium; MRT: Mental rotation test; RMET: Reading the mind in the eyes test; SNP: Single nucleotide polymorphism; SQ-R: Systemizing quotient, revised.

\section{Competing interests}

The authors declare they have no competing interests.

\section{Authors' contributions}

$\mathrm{BC}$ and SBC co-designed the study; WW and BC co-conducted the analysis, and SBC obtained funding for the study. All authors wrote the paper. All authors read and approved the final manuscript.

\section{Acknowledgements}

This study was funded by two project grants from Target Autism Genome, the Nancy Lurie Marks (NLM) Family Foundation, The Autism Research Trust, the Medical Research Council UK, the Wellcome Trust Sanger Centre, and the Max Planck Institute for Psycholinguistics. WW was funded by the Nehru Trust for Cambridge University, and the Cambridge Commonwealth Trust. BC was funded by the Medical Research Council UK. The study was conducted in association with the NIHR CLAHRC for Cambridgeshire and Peterborough. We are grateful to Robert Plomin, Frank Dudbridge, Lindsey Kent, and lan Craig for valuable discussions, and to Laura Murphy, Jonathan Breidbord, Allen Chan, Sylvia Lakatosova, Sally Wheelwright, Carrie Allison, Uma Mallya, Alex Politt, and the late Leena Peltonen for assistance at different stages of the project. Simon Fisher provided partial funding for the project. This study was submitted in part fulfillment of the degree of MPhil by WW to the University of Cambridge.

\section{Author details}

${ }^{1}$ Department of Psychiatry, Autism Research Centre, University of Cambridge, Cambridgeshire, UK. ${ }^{2}$ CLASS Clinic, Cambridgeshire and Peterborough NHS Foundation Trust (CPFT), Cambridgeshire, UK. ${ }^{3}$ Centre for Integrative Neuroscience and Neurodynamics, School of Psychology and Clinical Language Sciences, University of Reading, Reading, UK.

Received: 22 July 2013 Accepted: 31 October 2013

Published: 9 December 2013

\section{References}

1. American Psychiatric Association: Diagnostic and Statistical Manual of Mental Disorders: DSM-IV. 4th edition. Washington, DC: American Psychiatric Association; 1994.

2. Hallmayer J, Cleveland S, Torres A, Phillips J, Cohen B, Torigoe T, Miller J, Fedele A, Collins J, Smith K, Lotspeich L, Croen LA, Ozonoff S, Lajonchere C, Grether JK, Risch N: Genetic heritability and shared environmental factors among twin pairs with autism. Arch Gen Psychiatry 2011, 68:1095-1102.

3. Klei L, Sanders SJ, Murtha MT, Hus V, Lowe JK, Willsey AJ, Moreno-De-Luca D, Yu TW, Fombonne E, Geschwind D, Grice DE, Ledbetter DH, Lord C, Mane SM, Martin CL, Martin DM, Morrow EM, Walsh CA, Melhem NM, Chaste P, Sutcliffe JS, State MW, Cook EH, Roeder K, Devlin B: Common genetic variants, acting additively, are a major source of risk for autism. Mol Autism 2012, 3:9.

4. Baron-Cohen S, Scott FJ, Allison C, Williams J, Bolton P, Matthews FE, Brayne C: Prevalence of autism-spectrum conditions: UK school-based population study. Br J Psychiatry 2009, 194:500-509.

5. American Psychiatric Association: Task Force on DSM-IV: Diagnostic and Statistical Manual of Mental Disorders: DSM-IV-TR. 4th edition. Washington, DC: American Psychiatric Association; 2000.

6. Bourgeron T: A synaptic trek to autism. Curr Opin Neurobiol 2009, 19:231-234. 
7. Bill BR, Geschwind DH: Genetic advances in autism: heterogeneity and convergence on shared pathways. Curr Opin Genet Dev 2009, 19:271-278. 8. Persico AM, Napolioni V: Autism genetics. Behav Brain Res 2013. Epub. 9. Samaco RC, Hogart A, LaSalle JM: Epigenetic overlap in autism-spectrum neurodevelopmental disorders: MECP2 deficiency causes reduced expression of UBE3A and GABRB3. Hum Mol Genet 2005, 14:483-492.

10. Gaetz W, Bloy L, Wang DJ, Port RG, Blaskey L, Levy SE, Roberts TPL: GABA estimation in the brains of children on the autism spectrum: measurement precision and regional cortical variation. Neuroimage 2013, 13:00568-5.

11. Ma DQ, Whitehead PL, Menold MM, Martin ER, Ashley-Koch AE, Mei H, Ritchie MD, Delong GR, Abramson RK, Wright HH, Cuccaro ML, Hussman JP, Gilbert JR, Pericak-Vance MA: Identification of significant association and gene-gene interaction of GABA receptor subunit genes in autism. Am J Hum Genet 2005, 77:377-388.

12. Chakrabarti B, Dudbridge F, Kent L, Wheelwright S, Hill-Cawthorne G, Allison C, Banerjee-Basu S, Baron-Cohen S: Genes related to sex steroids, neural growth, and social-emotional behavior are associated with autistic traits, empathy, and Asperger syndrome. Autism Res 2009, 2:157-177.

13. Buxbaum JD, Silverman JM, Smith CJ, Greenberg DA, Kilifarski M, Reichert J, Cook EH, Fang $Y$, Song C-Y, Vitale R: Association between a GABRB3 polymorphism and autism. Mol Psychiatry 2002, 7:311-316.

14. Schroer RJ, Phelan MC, Michaelis RC, Crawford EC, Skinner SA, Cuccaro M, Simensen RJ, Bishop J, Skinner C, Fender D, Stevenson RE: Autism and maternally derived aberrations of chromosome 15q. Am J Med Genet 1998, 76:327-336.

15. Pizzarelli R, Cherubini E: Alterations of GABAergic signaling in autism spectrum disorders. Neural Plast 2011, 2011:297153.

16. Fatemi SH, Reutiman TJ, Folsom TD, Thuras PD: GABA(A) receptor downregulation in brains of subjects with autism. J Autism Dev Disord 2009, 39:223-230.

17. Cook EH, Courchesne RY, Cox NJ, Lord C, Gonen D, Guter SJ, Lincoln A, Nix K, Haas R, Leventhal BL, Courchesne E: Linkage-disequilibrium mapping of autistic disorder, with 15q11-13 markers. Am J Hum Genet 1998, 62:1077-1083.

18. Delahanty RJ, Kang JQ, Brune CW, Kistner EO, Courchesne E, Cox NJ, Cook EH Macdonald RL, Sutcliffe JS: Maternal transmission of a rare GABRB3 signal peptide variant is associated with autism. Mol Psychiatry 2011, 16:86-96.

19. Tavassoli T, Auyeung B, Murphy LC, Baron-Cohen S, Chakrabarti B: Variation in the autism candidate gene GABRB3 modulates tactile sensitivity in typically developing children. Mol Autism 2012, 3:6.

20. DeLorey TM, Sahbaie P, Hashemi E, Homanics GE, Clark JD: Gabrb3 gene deficient mice exhibit impaired social and exploratory behaviors, deficits in non-selective attention and hypoplasia of cerebellar vermal lobules: a potential model of autism spectrum disorder. Behav Brain Res 2008, 187:207-220.

21. Ben-Ari Y, Khazipov R, Leinekugel $X$, Caillard O, Gaiarsa JL: GABAA, NMDA and AMPA receptors: a developmentally regulated "ménage à trois". Trends Neurosci 1997, 20:523-529.

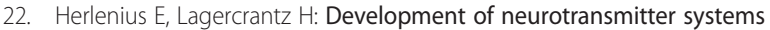
during critical periods. Exp Neurol 2004, 190(Suppl):S8-S21.

23. Woodbury-Smith MR, Robinson J, Wheelwright S, Baron-Cohen S: Screening adults for Asperger Syndrome using the AQ: a preliminary study of its diagnostic validity in clinical practice. J Autism Dev Disord 2005, 35:331-335.

24. Hoekstra RA, Bartels M, Verweij CJH, Boomsma Dl: Heritability of autistic traits in the general population. Arch Pediatr Adolesc Med 2007, 161:372-377.

25. Baron-Cohen S, Wheelwright S: The empathy quotient: an investigation of adults with Asperger syndrome or high functioning autism, and normal sex differences. J Autism Dev Disord 2004, 34:163-175.

26. Bons D, van den Broek E, Scheepers F, Herpers P, Rommelse N, Buitelaaar JK: Motor, emotional, and cognitive empathy in children and adolescents with autism spectrum disorder and conduct disorder. J Abnorm Child Psychol 2013, 41:425-443.

27. Emde RN, Plomin R, Robinson JA, Corley R, DeFries J, Fulker DW, Reznick JS, Campos J, Kagan J, Zahn-Waxler C: Temperament, emotion, and cognition at fourteen months: the MacArthur Longitudinal Twin Study. Child Dev 1992, 63:1437-1455.

28. Knafo A, Zahn-Waxler C, Van Hulle C, Robinson JL, Rhee SH: The developmental origins of a disposition toward empathy: genetic and environmental contributions. Emotion 2008, 8:737-752.

29. Baron-Cohen S, Richler J, Bisarya D, Gurunathan N, Wheelwright S: The systemizing quotient: an investigation of adults with Asperger syndrome or high-functioning autism, and normal sex differences. Philos Trans $R$ SoC Lond B Biol Sci 2003, 358:361-374.

30. Baron-Cohen S: Autism: research into causes and intervention. Pediatr Rehabil 2004, 7:73-78.

31. Wheelwright S, Baron-Cohen S, Goldenfeld N, Delaney J, Fine D, Smith R, Weil L, Wakabayashi A: Predicting Autism Spectrum Quotient (AQ) from the Systemizing Quotient-Revised (SQ-R) and Empathy Quotient (EQ). Brain Res 2006, 1079:47-56.

32. Baron-Cohen S, Wheelwright S, Hill J, Raste Y, Plumb I: The "Reading the Mind in the Eyes" Test revised version: a study with normal adults, and adults with Asperger syndrome or high-functioning autism. J Child Psychol Psychiatry 2001, 42:241-251.

33. Losh M, Piven J: Social-cognition and the broad autism phenotype: identifying genetically meaningful phenotypes. J Child Psychol Psychiatry 2007, 48:105-112.

34. Jolliffe T, Baron-Cohen S: Are people with autism and Asperger syndrome faster than normal on the Embedded Figures Test? J Child Psychol Psychiatry 1997, 38:527-534.

35. Baron-Cohen S, Ring H, Chitnis X, Wheelwright S, Gregory L, Williams S, Brammer M, Bullmore E: fMRI of parents of children with Asperger Syndrome: a pilot study. Brain Cogn 2006, 61:122-130.

36. Falter CM, Plaisted KC, Davis G: Visuo-spatial processing in autism-testing the predictions of extreme male brain theory. J Autism Dev Disord 2008, 38:507-515

37. McGee MG: Handedness and mental rotation. Percept Mot Skills 1978, 47:641-642

38. Baron-Cohen S, Wheelwright S, Skinner R, Martin J, Clubley E: The autism-spectrum quotient (AQ): evidence from Asperger syndrome/ high-functioning autism, males and females, scientists and mathematicians. J Autism Dev Disord 2001, 31:5-17.

39. Purcell S, Neale B, Todd-Brown K, Thomas L, Ferreira MAR, Bender D, Maller J, Sklar P, de Bakker PIW, Daly MJ, Sham PC: PLINK: a tool set for whole-genome association and population-based linkage analyses. Am J Hum Genet 2007, 81:559-575.

40. Nyholt DR: A simple correction for multiple testing for single-nucleotide polymorphisms in linkage disequilibrium with each other. Am J Hum Genet 2004, 74:765-769.

41. Johnson AD, Handsaker RE, Pulit SL, Nizzari MM, O'Donnell CJ, de Bakker PIW SNAP: a web-based tool for identification and annotation of proxy SNPs using HapMap. Bioinformatics 2008, 24:2938-2939.

42. Barrett JC, Fry B, Maller J, Daly MJ: Haploview: analysis and visualization of LD and haplotype maps. Bioinformatics 2005 21:263-265.

43. Lee $\mathrm{PH}$, Shatkay $\mathrm{H}$ : An integrative scoring system for ranking SNPs by their potential deleterious effects. Bioinformatics 2009, 25:1048-1055.

44. Yuan H-Y, Chiou J-J, Tseng W-H, Liu C-H, Liu C-K, Lin Y-J, Wang H-H, Yao A, Chen Y-T, Hsu C-N: FASTSNP: an always up-to-date and extendable service for SNP function analysis and prioritization. Nucleic Acids Res 2006, 34(Web Server issue):W635-W641.

45. Chelala C, Khan A, Lemoine NR: SNPnexus: a web database for functiona annotation of newly discovered and public domain single nucleotide polymorphisms. Bioinformatics 2008, 25:655-661.

46. Ward LD, Kellis M: HaploReg: a resource for exploring chromatin states, conservation, and regulatory motif alterations within sets of genetically linked variants. Nucleic Acids Res 2012, 40(Database issue):D930-D934.

47. Kent WJ, Sugnet CW, Furey TS, Roskin KM, Pringle TH, Zahler AM, Haussler D: The human genome browser at UCSC. Genome Res 2002, 12:996-1006.

48. James SJ, Shpyleva S, Melnyk S, Pavliv O, Pogribny IP: Complex epigenetic regulation of engrailed-2 (EN-2) homeobox gene in the autism cerebellum. Transl Psychiatry 2013, 3:e232.

49. Kim SA, Kim JH, Park M, Cho $H_{\text {, }}$ Yoo HJ: Association of GABRB3 polymorphisms with autism spectrum disorders in Korean trios. Neuropsychobiology 2006, 54:160-165.

50. Gharani N, Benayed R, Mancuso V, Brzustowicz LM, Millonig JH: Association of the homeobox transcription factor, ENGRAILED 2, 3, with autism spectrum disorder. Mol Psychiatry 2004, 9:474-484.

51. Benayed R, Gharani N, Rossman I, Mancuso V, Lazar G, Kamdar S, Bruse SE, Tischfield S, Smith BJ, Zimmerman RA, Dicicco-Bloom E, Brzustowicz LM, Millonig JH: Support for the homeobox transcription factor gene ENGRAILED 2 as an autism spectrum disorder susceptibility locus. Am J Hum Genet 2005, 77:851-868. 
52. Wang L, Jia M, Yue W, Tang F, Qu M, Ruan Y, Lu T, Zhang H, Yan H, Liu J, Guo Y, Zhang J, Yang X, Zhang D: Association of the ENGRAILED 2 (EN2) gene with autism in Chinese Han population. Am J Med Genet B Neuropsychiatr Genet 2008, 147B:434-438.

53. Kiefer JC: Back to basics: Sox genes. Dev Dyn 2007, 236:2356-2366.

54. Ghaziuddin M, Mountain-Kimchi K: Defining the intellectual profile of Asperger syndrome: comparison with high-functioning autism. J Autism Dev Disord 2004, 34:279-284.

55. Hoekstra RA, Happé F, Baron-Cohen S, Ronald A: Limited genetic covariance between autistic traits and intelligence: findings from a longitudinal twin study. Am J Med Genet B Neuropsychiatr Genet 2010, 153B:994-1007.

doi:10.1186/2040-2392-4-48

Cite this article as: Warrier et al:: Genetic variation in GABRB3 is associated with Asperger syndrome and multiple endophenotypes relevant to autism. Molecular Autism 2013 4:48.

\section{Submit your next manuscript to BioMed Central and take full advantage of:}

- Convenient online submission

- Thorough peer review

- No space constraints or color figure charges

- Immediate publication on acceptance

- Inclusion in PubMed, CAS, Scopus and Google Scholar

- Research which is freely available for redistribution 